\title{
Lateral Pedicle Graft Procedure for the Treatment of Isolated Tooth Recession- A Case Report
}

\author{
Neelam Rana ${ }^{1}$, Sachit Anand Arora $^{2}$, Rupali Kalsi ${ }^{3}$, Kumar Saurav $^{4}$ \\ Department of Periodontics, I.T.S Dental College, Hospital and Research Centre, Knowledge Park-III, \\ Greater Noida, Uttar Pradesh, 20130, India \\ Corresponding Author: Neelam Rana
}

\begin{abstract}
Gingival recession is defined as the apical migration of the gingival margin from its physiologic level which further causes pathologic exposure of root surfaces. Mucogingival esthetic surgery includes the treatment of mucogingival esthetic alterations. The Mucogingival problems may be caused by gingival recession which is often associated with non-carious cervical lesion or by root caries or by altered passive eruption. Root coverage is achieved by many procedures like free gingival autografts, pedicle graft and connective tissue grafts. Recession can be treated by surgical or non-surgical means. Surgical procedure includes various methods of increasing the width of keratinized tissue such as frenectomy in case of high frenal attachment $\&$ root coverage procedures and nonsurgical approach include-restorations, crowns, veneers and gingival masks. Lateral displaced pedicle flap was first described by Grupe and Warren in 1956 which was indicated in area to cover isolated apical migration of gingiva, \& denuded root surfaces that have adequate amount of donor tissue adjacent to the recipient site. In few clinical studies it is seen that the success rate with Lateral pedicle graft is $70 \%$, it is preferred over free gingival autografts in narrow and shallow gingival defects. This case report present the predictability of root coverage by using Lateral pedicle grafts in single tooth narrow and shallow gingival recession.
\end{abstract}

Key words: Lateral pedicle graft, Gingival recession, Attachment loss, Graft

\section{INTRODUCTION}

Mucogingival esthetic surgery includes the treatment of mucogingival esthetic alterations and restoration. The apical shift of the gingival epithelium leading to the exposure of the root surface is termed as Gingival Recession. There are various etiological factors of gingival recession and the treatment of it should eliminate the etiological factors followed by achieving the root coverage of the denuded surface. Recession may also lead to accumulation of plaque and calculus, loss of aesthetics, root caries, abrasion of the root surfaces and hypersensitivity. ${ }^{1}$ There are many surgical treatment modalities used for the management of gingival recessions and each technique has their own advantages and limitations over others. ${ }^{2}$ Following surgical procedures are indicated for treatment of gingival recessions, they include $^{3}$

a. Lateral pedicle graft

b. Double papilla flap

c. Oblique rotated flap

d. Coronally repositioned flap

e. Semilunar coronally repositioned flap.

Grupe and Warren ${ }^{5}$ was the first to describe the Lateral Sliding Flap in year 1956. For single isolated tooth root coverage where the amount of adequate soft tissue and underlying bone is present at the donor site makes the prognosis of lateral sliding flap good ${ }^{4}$. The advantages of pedicle grafts when compared to autografts is one surgical site with preservation of 
blood supply to pedicle graft which will prevent graft necrosis and rejection which results in post operative colour harmony with adjacent gingival tissues ${ }^{10}$.

\section{CASE REPORT}

A 28 years old female patient reported to the outpatient Department of Periodontics and implantology with the

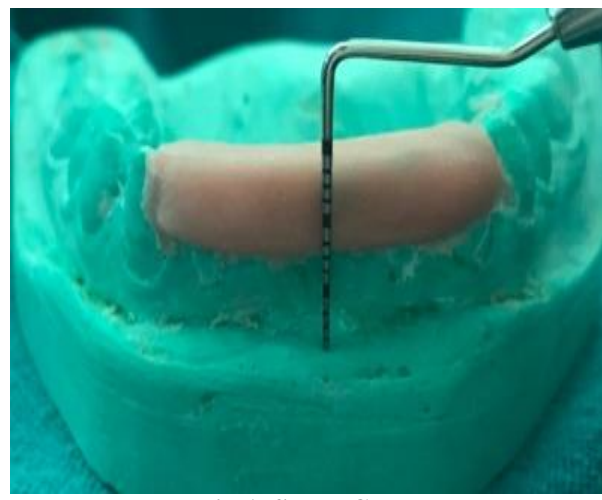

Fig A-Study Cast

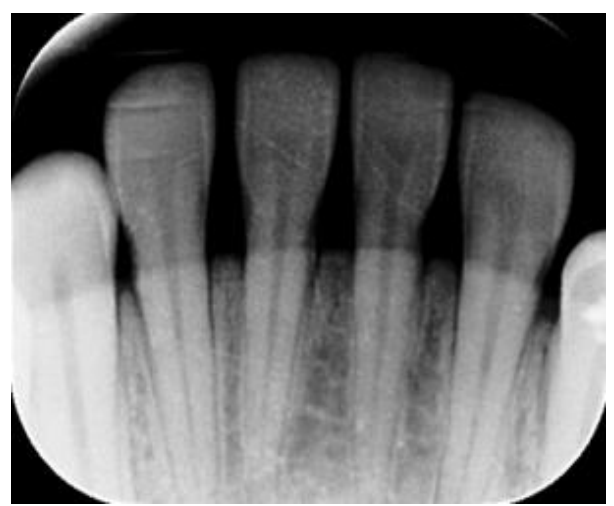

Fig C-IOPA chief complaint of mild hypersensitivity and lowering of gums in her lower front tooth region for past 3 months. The patient reported no previous medical and dental history. On examination Miller's Class I recession was elicited in 31 (Figure-B) with an adequate zone of AG (Figure-c). Informed consent was obtained from the patient before surgical procedure.

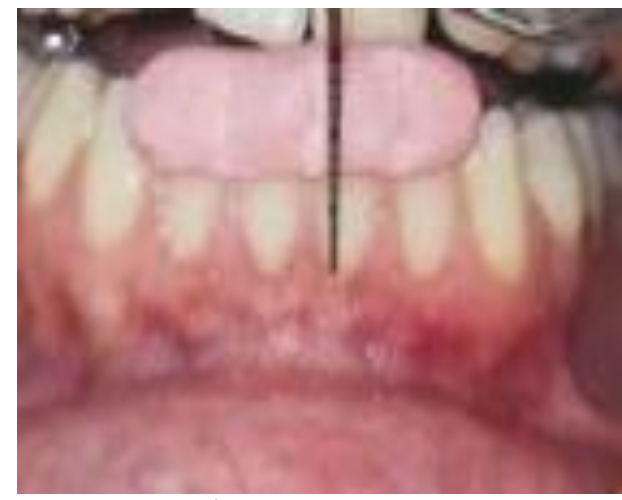

Fig B-Pocket Depth

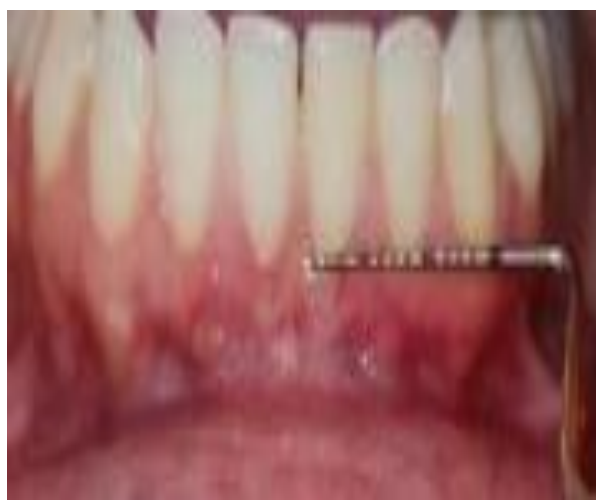

Fig D-Width of attached gingiva

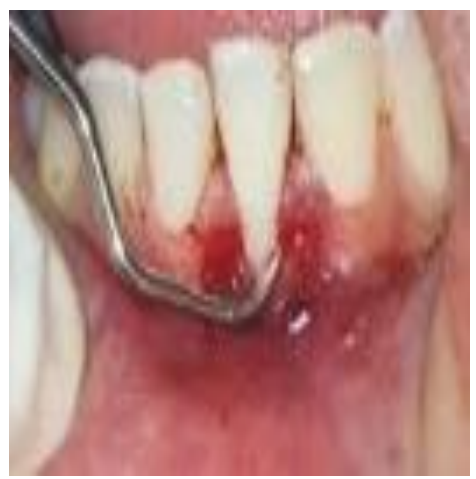

Fig E-Root Planing

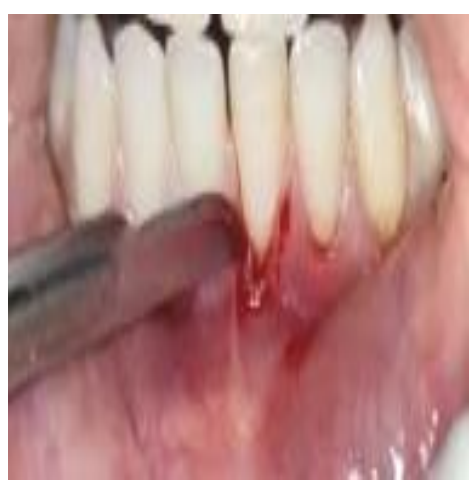

Fig F- Horizontal and vertical incision

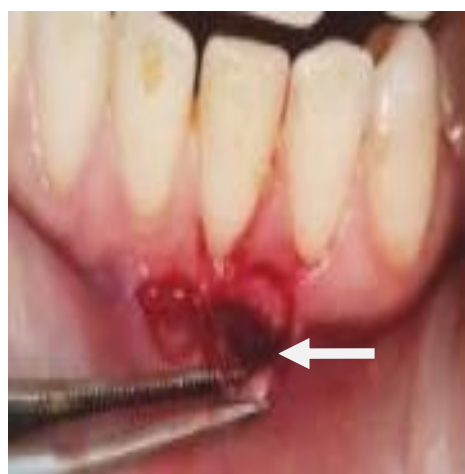

Fig G- Split thickness flap
At baseline, clinical parameters like pocket probing depth (PPD) (Fig-B) and width of attached gingival were recorded (Fig-D). Mucogingival junction (MGJ) was located by visual method. Pocket depth was measured using UNC-15 probe distance from gingival margin to the base of the sulcus. Intraoral periapical radiograph of this region suggests no bone loss (Fig-C); hence case was diagnosed to be a case of Miller's class I. Treatment planning was done. Phase I therapy (Etiotrophic Phase) 
included scaling and root planning followed by oral hygiene instructions. Patient was recalled after 2 weeks of phase I therapy and

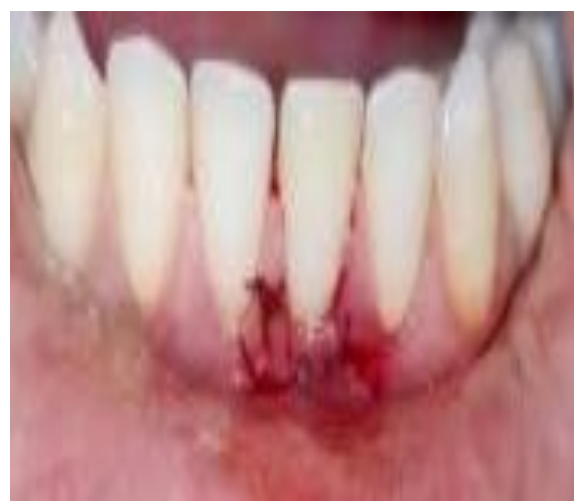

Fig H-Interrupted Suture

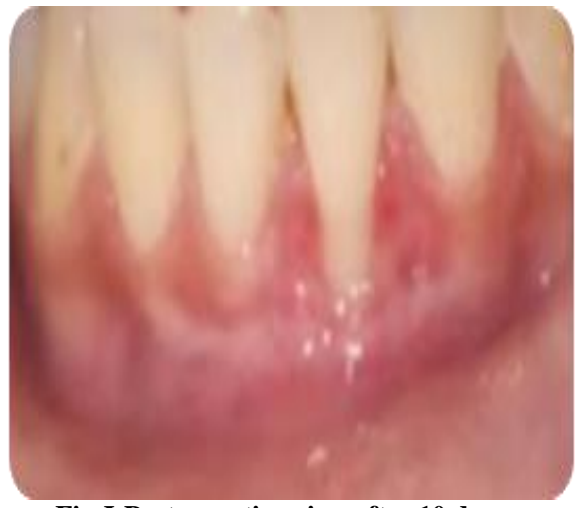

Fig J-Postoperative view after 10 days

\section{Surgical Procedure}

The lateral pedicle graft procedure was performed according to the protocol given by Grupe and Warren in 1965.Firstly, Local anesthesia using $2 \%$ lignocaine hydrochloride with 1:2,00,000 epinephrine was administered. With the help of No. 15 blade, De-epithelization of the gingival margin around the exposed root is done to provide a recipient bed for the repositioned tissue. After this, root surface is thoroughly curetted to remove the granulation tissue present along the incised edge along with use of EDTA (root biomodification) to achieve smooth surface.

With a no.15 blade, Two vertical incisions were made, one at distal line angle of 31 and other at mesial line angle of 32 . Two vertical incisions along with horizontal incisions were made, and then it was extended apical to the mucosal tissue to permit the mobility of flap. A split-thickness flap is then raised (Figure-G). The split- then surgical phase i.e. LPG procedure was carried out.

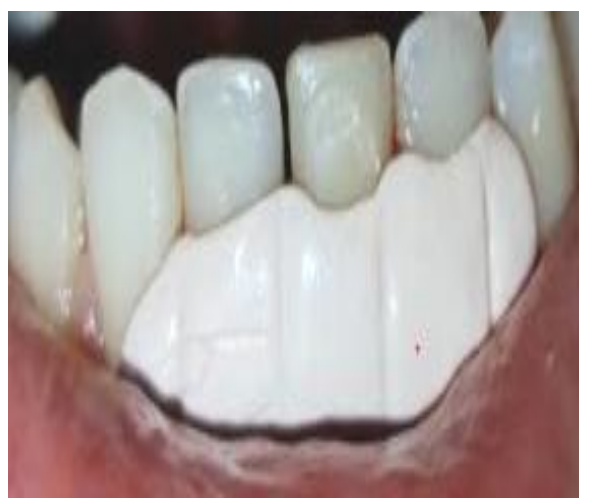

Fig I-COE Pack placed

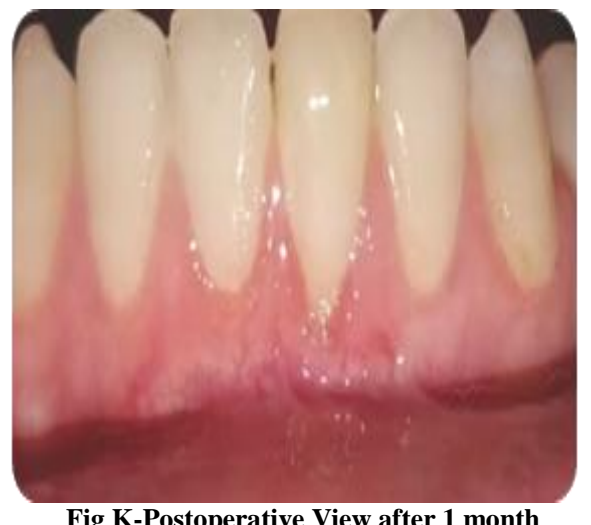

Fig K-Postoperative View after 1 month

thickness pedicle flap is then repositioned over the recipient site with interrupted and sling sutures After suturing, gentle pressure is applied for few minutes with help of moist gauze to minimize clot underneath the pedicle flap. A cut back releasing incision was made to ensure that the flap is free of tension is free enough to permit movement to the recipient site [Figure-F]. Pedicle flap was positioned $1 \mathrm{~mm}$ coronal to cementoenamel junction of 31 and sutured by 4-0 silk sutures [Figure-H]. The area was protected with Coe-Pack [Figure-I].

\section{Postoperative care}

The patient was instructed regarding post operative care of the surgical site. She was advised not to brush on the operated area and use of chlorhexidine gluconate mouth wash of $0.12 \%$ twice daily. Patient was also kept on a course of Amoxicillin (500mg thrice daily for 5 consecutive days) and Aceclofenac (100mg thrice daily for 3 
days). Sutures were removed 10days after surgery and the examination of surgical site showed complete coverage (Fig.) of the root surface. The patient was recalled after 1 month. The pedicle graft after 1-month showed firm attachment to the root on surgical site.

\section{OUTCOME}

Pre and post-operative clinical measurement was compared. Postoperatively, there was a firm attachment of coverage on denuded root surface

\section{DISCUSSION}

Gingival recession usually results in poor aesthetics, pain and sensitivity, root caries. There are several surgical techniques are to manage gingival recession defects including root coverage procedures, increasing the width of keratinized tissue ${ }^{1}$.

Root coverage procedures have become a important treatment modality for gingival recession ${ }^{2}$. The success rate and prognosis of root coverage procedures depends on several factors like elimination or control of etiological factors, interproximal bone level, and the choice of best coverage procedure based on the clinical situation. In the present case, we chose Lateral pedicle graft technique because of the good periodontal condition of the neighboring tooth with adequate keratinized gingival and normal bone height ${ }^{5}$.

Advantages of using lateral pedicle graft over the root coverage procedure are that it requires only a single surgical site, with no separate donor site. Disadvantage of lateral pedicle graft is possible bone loss and gingival recession on the donor site. According to Guinard and Caffesse ${ }^{8}$ an average of $1 \mathrm{~mm}$ of post-operative gingival recession on the adjacent donor site.

Therefore, LPG is contraindicated where the width and thickness of keratinized gingival of the donor tissue is inadequate. Many modification methods of Grupe and Warren $^{5}$ have been developed to avoid donor site gingival recession. Staffileno ${ }^{2}$ stated that the use of partial thickness flap avoid the recession at donor site. Grupe ${ }^{6}$ reported a modified technique to preserve the marginal gingival by making submarginal incision at donor site. Whereas, Pteifer $\mathrm{Js}^{4}$ et al demonstrated the method of full and partial thickness pedicle flap. Moawia M Kassab et al in 2000 reported that use of EDTA results in significant improvement postoperative in depth of recession, root coverage, and clinical attachment levels.

\section{CONCLUSION}

Lateral pedicle graft is a reliable procedure for isolated denuded root surface. LPG is simple surgical procedure when it is compared with other mucogingival procedure such as free gingival graft. It offers considerable advantage such as one surgical site and good vascularity which help in uptake of graft. The predictable outcome of treatment in case of single tooth or isolated gingival recession is excellent and complete coverage can be expected for Miller's Class-I and Class-II conditions using lateral pedicle grafts and also colour matching with the adjacent tissues.

Acknowledgement: None

Conflict of Interest: None

\section{Source of Funding: None}

\section{REFERENCES}

1. Woofer C: The prevalence and etiology of gingival recession, periodont Abstr 17:43, 1969.

2. Staffileno H. Management of gingival recession and root exposure problems associated with periodontal disease. Compend Contin Educ Dent 1964; November: 111-120.

3. Bahat O, Handelsman M. Periodontal reconstructive flaps classification and surgical considerations. Int. J Periodontics Restorative Dent 1991; 11:481-487.

4. Pteifer Js, Heller R. Histologic evaluation of full and partial thickness laterally repositioned flaps-A pilot study. J periodontal 197; 42:331 
5. Grupe HE, Warren RF Jr: Repair of gingival defects by a sliding flap operation, J periodontal 27:923, 1956.

6. Grupe HE: Modified technique for sliding flap operation. J periodontal 37:491, 1966.

7. Guinard EA, Caffesse RG. Treatment of localized gingival recessions: Part I. Lateral sliding flap. J Periodontol. 1978; 49: 351356.

8. Miller PD Jr: A classification of marginal gingival recession, Int $\mathrm{J}$ periodont Restor Dent 5:9, 1985.
9. Sullivan HC, Atkins JC: Free Autogenous gingival graft 111. Utilization of graft in the treatment of gingival recession Periodontics 6:152, 1968 .

How to cite this article: Rana N, Arora SA, Kalsi R et.al. Lateral pedicle graft procedure for the treatment of isolated tooth recession- a case report. International Journal of Science \& Healthcare Research. 2021; 6(2): 9-13. DOI: https://doi.org/10.52403/ijshr.20210402 